\title{
The distribution of early-type stars in the Mon-CMa-Pup-Vel region of the Milky Way
}

\author{
N. T. Kaltcheva \\ Department of Physics and Astronomy, University of Wisconsin Oshkosh, 800 Algoma Blvd., \\ Oshkosh, WI 54901, USA \\ email: kaltchev@uwosh.edu
}

\begin{abstract}
An overall uvby $\beta$ photometric survey of the structure of the star-forming fields located toward the Monoceros-Canis Major-Puppis-Vela region of the Milky Way is presented.
\end{abstract}

Keywords. stars: distances, open clusters and associations: general, Galaxy: structure

\section{Introduction}

Unlike the external galaxies where the star-forming fields are generally evident from direct imaging, in our own Galaxy the spiral arms are strung out along the line of sight, leading to the superposition and mixing of different star-forming complexes in the sky. Thus, the study of the structure of the star-forming fields of the MW is grounded in distance determinations of young stellar tracers. At present, very few Galactic star-forming regions benefit from numerous stellar studies, capable to provide sufficient knowledge about the causal relationship between different stellar populations. The precise mapping of the Mon-CMa-Pup-Vel field presented here provides the basis for a better understanding of how the massive stars dominate the structure and evolution of their environments. The far-reaching goal is to correlate the locations of the optical spiral tracers and the interstellar material.

\section{Discussion}

In this context, the quantity, quality and completeness of the observational data and their interpretation play a critical role in gaining a satisfactory degree of understanding. The present study is based on uvby $\beta$ photometry of O- and B-type stars. This photometric system is arguably better suited to the study of individual stars and their groupings in terms of stellar luminosity than any other photometric system in wide use. It provides accurate stellar distances allowing not only to delineate the general Galactic spiral structure, but also to follow arm splitting and branching. Based on a complete magnitude-limited sample of O-B9 stars, the stellar content, the distribution of the interstellar absorption, the spatial stellar distribution, and metalicity and age variations across the field are analyzed. A homogeneous distance scale is established, implying that the prominent young structures studied are closer to the Sun than was previously thought (Kaltcheva \& Hilditch, 2000, Kaltcheva 2006).

\section{References}

Kaltcheva, N.T. \& Hilditch, R. 2000, MNRAS 312, 753

Kaltcheva, N.T. 2006, $A N$ submitted 